\title{
High-resolution study of the young stellar objects in Mon R2 IRS 3
}

\author{
T. Preibisch ${ }^{1}$, Y. Y. Balega ${ }^{2}$, D. Schert ${ }^{1}$, and G. Weigelt ${ }^{1}$ \\ 1 Max-Planck-Institut für Radioastronomie, Auf dem Hügel 69, 53121 Bonn, Germany \\ 2 Special Astrophysical Observatory, Nizhnij Arkhyz, Zelenchuk region, Karachai-Cherkesia, 357147, Russia
}

\author{
Received 22 May 2002 / Accepted 21 June 2002
}

\begin{abstract}
We present a bispectrum speckle interferometry study of the embedded young stellar objects in Mon R2 IRS 3 in the near-infrared $H$ and $K$ bands. Our images with a resolution of 75 mas ( $\widehat{=} 62 \mathrm{AU}$ ) show a close triple system surrounded by strong diffuse nebulosity and three additional infrared sources ( $K$ magnitudes $\sim 11.8-13.7$ ) within 3 " of the brightest object IRS 3 A $(K \sim 7.9)$. We use HST/NICMOS archive images to derive near-infrared photometry for the sources and estimate the stellar masses of the three brightest objects IRS $3 \mathrm{~A}, \mathrm{~B}$, and $\mathrm{C}$ to be in the range $\sim 5-15 M_{\odot}$. IRS 3 A is surrounded by a bipolar nebula (position angle $\sim 30^{\circ}$ ), suggesting it to be embedded in a thick circumstellar disk or a torus with polar cavities. IRS 3 B shows a remarkable jet-like emission feature pointing towards the north-east (position angle $50^{\circ}$ ). This feature consists of at least three individual knots with projected separations of 130, 230, and 290 mas (110, 190, and 240 AU) from IRS 3 B, which are much brighter in the $K$ band than in the $H$ band. This strongly indicates outflow activity from IRS 3 B, which is therefore probably the source of the compact high velocity molecular outflow reported from Mon R2 IRS 3 . We also analyze Chandra X-ray archive data for the Mon R2 region and find IRS 3 A and IRS 3 C to be sources of hard $(3-10 \mathrm{keV})$ and variable $\mathrm{X}$-ray emission, suggesting plasma temperatures of at least $50 \times 10^{6} \mathrm{~K}$. The similarity of the X-ray properties to those of Class I protostars suggests magnetic interaction between the protostars and their circumstellar disks to be the origin of the $\mathrm{X}$-ray emission; this provides indirect evidence for the presence of circumstellar disks in the intermediate- to high-mass young stellar objects IRS 3 A and IRS 3 C.
\end{abstract}

Key words. techniques: interferometric - stars: individual: Mon R2 IRS 3 - stars: formation - stars: X-rays stars: winds, outflows

\section{Introduction}

The Mon R2 cluster is one of the closest $(D \sim 830 \mathrm{pc}$; Herbst \& Racine 1976) massive star forming regions that is still embedded in its natal molecular cloud. Following the initial detection of seven bright infrared sources by Beckwith et al. (1976), studies by Aspin \& Walther (1990), Howard et al. (1994) and Carpenter et al. (1997; C97 hereafter) demonstrated the existence of an extensive stellar population in the Mon R2 molecular cloud. The cluster contains several hundred stars which are obscured by an average visual extinction of $33 \mathrm{mag}$ (C97). The brightest infrared sources in the Mon R2 region are IRS 1, IRS 2, and IRS 3; their luminosities, determined with detailed radiative transfer modeling by Henning et al. (1992), are $3000 L_{\odot}, 6500 L_{\odot}$, and $14000 L_{\odot}$, respectively. IRS 1 is the ionizing source of a compact HII region and its radio flux suggests it to be a ZAMS B0 star (Massi et al. 1985). IRS 2 illuminates the prominent ring nebulosity in the central part of the star forming region. IRS 3 , though the brightest infrared source and probably the most luminous young stellar object (YSO) in the cloud, is optically invisible and has no associated

Send offprint requests to: T. Preibisch,

e-mail: preib@mpifr-bonn.mpg.de
HII region (see Simon et al. 1981); this probably reflects the very early evolutionary stage of IRS 3 , which apparently has not yet developed an HII region.

The Mon R2 star forming region contains a large molecular outflow, which extends over $25^{\prime}$ ( $\sim 6 \mathrm{pc}$ ) and is extremely massive $\left(M \sim 200 M_{\odot}\right)$. On large scales, the flow shows a high degree of collimation (Wolf et al. 1990), but the central redand blueshifted high-velocity components are spatially mixed and poorly collimated. The outflow therefore seems to consist of two distinct pairs of bipolar lobes which partially overlap and whose major axes are roughly orthogonal (Meyers-Rice \& Lada 1991). The two bipolar lobe systems are either two separate outflows from separate sources, or two episodes of outflow activity. The source of the outflow system has not yet been convincingly identified. Giannakopoulou et al. (1997) found that the highest velocity blueshifted gas is associated with IRS 3 , but there is no evidence of a bipolar distribution of gas around IRS 3. This may be understood by assuming that IRS 3 is driving the compact, unresolved outflow, whereas the source of the larger outflow is no longer active.

First sub-arcsecond resolution infrared imaging by Beckwith et al. (1976) indicated IRS 3 to be a double source; this was later confirmed by McCarthy (1982), who derived 
Table 1. Observational parameters. $N_{\mathrm{T}}$ and $N_{\mathrm{R}}$ are the numbers of Mon R2 speckle interferograms and reference-star speckle interferograms, respectively. $T$ is the exposure time per frame, $S$ is the seeing $(F W H M)$. In the last column, the name of the reference stars are given.

\begin{tabular}{ccccccl}
\hline \hline$\lambda$ & Date & $N_{\mathrm{T}}$ & $N_{\mathrm{R}}$ & $\begin{array}{c}T \\
{[\mathrm{~ms}]}\end{array}$ & $\begin{array}{c}S \\
{\left[{ }^{\prime}\right]}\end{array}$ & Ref. star \\
\hline$K$ & 13.10 .2000 & 782 & 696 & 160 & 1.26 & HIP 30262 \\
& 15.10 .2000 & 115 & 517 & 240 & 1.48 & HIP 30262 \\
& & 115 & 522 & 240 & 1.46 & HIP 30262 \\
& 10.3 .2001 & 275 & 607 & 240 & 1.18 & HD 42827 \\
& & 280 & 602 & 240 & 1.18 & HD 42827 \\
& & 278 & 617 & 240 & 1.22 & HD 42827 \\
& 11.3 .2001 & 497 & 723 & 240 & 2.24 & HD 42827 \\
& 13.3 .2001 & 365 & 811 & 162 & 2.14 & HD 42827 \\
& & 340 & 813 & 162 & 2.02 & HD 42827 \\
& & 365 & 810 & 162 & 1.62 & HD 42827 \\
& & 364 & 811 & 162 & 1.66 & HD 42827 \\
& & 366 & 812 & 162 & 1.64 & HD 42827 \\
\hline$H$ & 10.3 .2001 & 672 & 608 & 360 & 1.50 & HD 42827 \\
\hline
\end{tabular}

a separation of $0.87^{\prime \prime}$ at a position angle of $13.5^{\circ}$. A speckle interferometric imaging study of IRS 3 in the near infrared $K(2.2 \mu \mathrm{m})$ and $L^{\prime}(3.8 \mu \mathrm{m})$ bands and at $4.8 \mu \mathrm{m}$ was presented by Koresko et al. (1993). In this study IRS 3 was clearly resolved as a $0.87^{\prime \prime}$ separation binary system. A bright conical nebula was found extending south from the southern component, and a previously unknown pointlike source was detected $0.37^{\prime \prime}$ east of the northern component in the $K$ band, but not at longer wavelengths. Further near-infrared speckle observations of IRS 3 have been obtained by Alvarez \& Hoare (2002).

\section{Observations and data reduction}

\subsection{Bispectrum speckle interferometry}

The speckle interferograms were recorded with the $6 \mathrm{~m}$ SAO telescope in October 2000 and March 2001 (see Table 1). The detector of our speckle camera was a Rockwell HAWAII array detector. The observations were made through $K$ band and $H$ band filters with center wavelength/FWHM bandwidth of $2.12 \mu \mathrm{m} / 0.21 \mu \mathrm{m}$ and $1.65 \mu \mathrm{m} / 0.32 \mu \mathrm{m}$. The pixel sizes of the $K$ band and $H$ band observations were 27.0 mas and 20.1 mas, respectively. The observational parameters are shown in Table 1 . The field-of-view is $5.4^{\prime \prime} \times 5.4^{\prime \prime}$ for both the $K$ band and the $H$ band observations.

Diffraction-limited images were reconstructed using the bispectrum speckle interferometry method (Weigelt 1977; Lohmann et al. 1983; Hofmann \& Weigelt 1986). The object power spectrum was determined with the speckle interferometry method (Labeyrie 1970). Speckle interferograms of unresolved single stars were recorded just before and after the object and served as reference stars for the determination of the speckle transfer function. The $K$ band image (Fig. 1) is diffraction-limited with a resolution of 75 mas, the $H$ band image was reconstructed with the same resolution. The dynamic range is $\sim 7$ mag for our $K$ band image and $\sim 4$ mag for our $H$ band image, respectively.

\subsection{HST NICMOS archive data}

The HST data archive contains numerous near-infrared NICMOS images covering different parts of the Mon R2 region. We retrieved and investigated calibrated NICMOS-2 images in the filters 110W $(\sim J)$, 160W $(\sim H)$, and 207M $(\sim K)$, which contain IRS 3 in their field-of-view. The data were obtained in December 1997 with exposure times of $256 \mathrm{~s}$ (110W), $288 \mathrm{~s}(160 \mathrm{~W}), 144 \mathrm{~s}(207 \mathrm{M})$. The diffraction limited resolution of the HST is 95 mas, 137 mas, and 180 mas in the $J, H$, and $K$ band respectively. However, due to the relatively large pixel size (75 mas) of the NICMOS-2 camera and the off-center position $^{1}$ of IRS 3 , the actual spatial resolution of these data is significantly lower, yielding $F W H M$ sizes of $\sim 160-180$ mas for point-like sources near IRS 3.

\subsection{Archival Chandra X-ray data}

From the Chandra X-ray data archive we retrieved the data of a deep observation of the Mon R2 star forming region. The observation (OBSID: 1882; PI: Koyama) was performed in the period December 2-4 2000 and has a total exposure time of 96838 seconds (nearly 27 hours). The X-ray image was obtained with the Advanced CCD Imaging Spectrometer ACIS-I, which gives a field of view of $17^{\prime} \times 17^{\prime}$ with a pixel size of $0.5^{\prime \prime}$. ACIS is sensitive to X-ray photons in the energy range $0.2-$ $10 \mathrm{keV}$, and provides an energy resolution of $E / \Delta E \sim 20-50$. The general results of this observation are reported in Kohno et al. (2002). Our analysis described in Sect. 4 is restricted to the sources in the immediate vicinity of IRS 3.

\section{Near-infrared results}

Figure 1 shows our $H$ and $K$ band speckle images of Mon R2 IRS 3 and a $H+K$ color composite image. The speckle images reveal six point-like sources in the field of view and show extended diffuse nebulosity.

\subsection{Near-infrared sources in Mon R2 IRS 3}

The three bright star-like sources in the center are the objects resolved for the first time by Koresko et al. (1993). There seems to be some confusion in the literature about the naming of these sources: While Koresko et al. (1993) use the names IRS 3 S, IRS 3 N, and IRS 3 NE, C97 denote the two brightest components IRS 3 SW and IRS 3 NE. We therefore will denote the six objects visible in our image as IRS 3 A, B, C, D, E, and F in order of decreasing $K$ band brightness. In our nomenclature, IRS 3 A corresponds to IRS $3 \mathrm{~S}$ in the convention of Koresko et al. (1993), IRS 3 B corresponds to IRS 3 N, and IRS 3 C to IRS 3 NE. IRS 3 E is source \# 278 in C97.

\footnotetext{
${ }^{1}$ IRS 3 is located about 4 " west of the edge of the chip.
} 

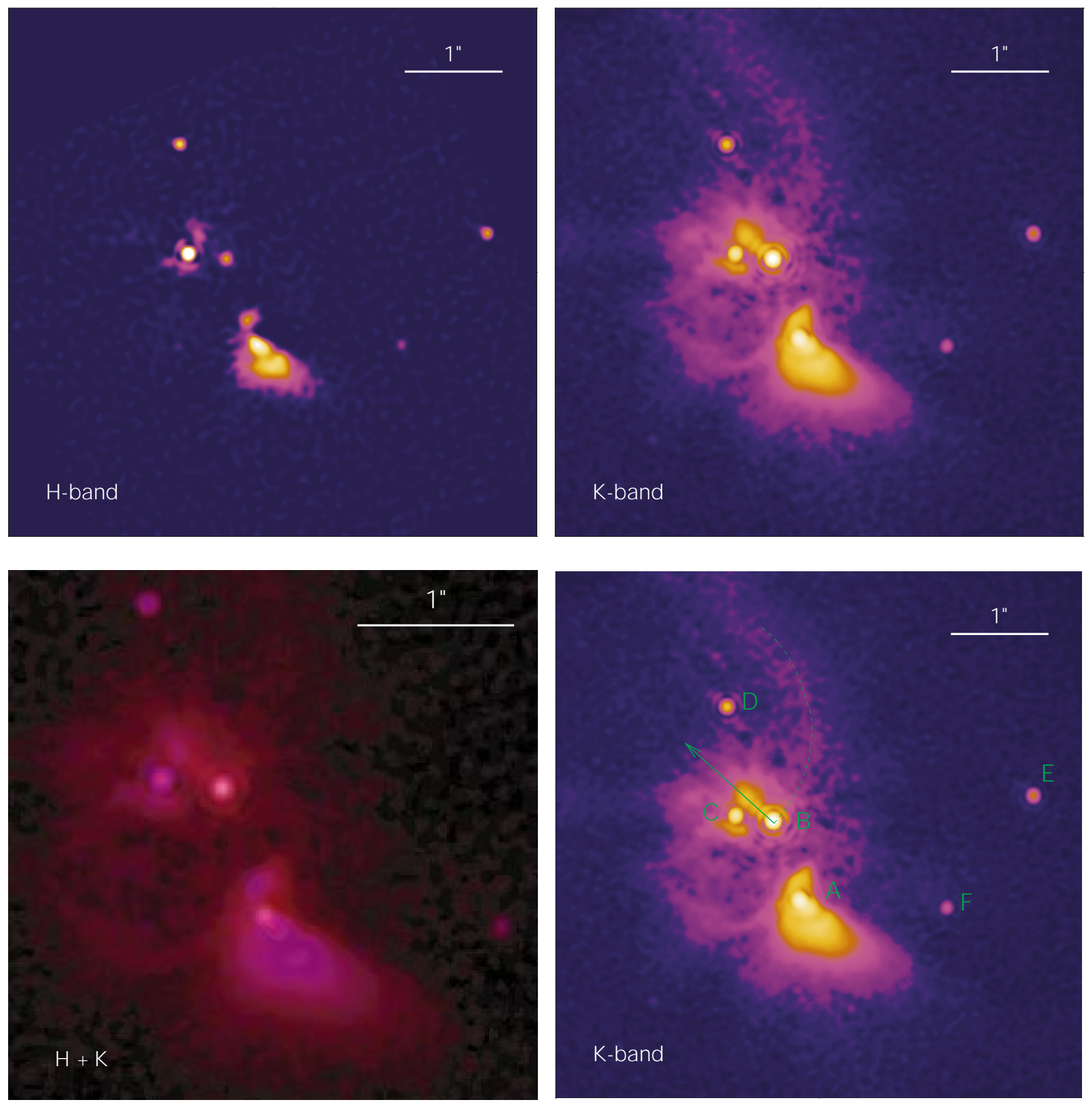

Fig. 1. Images of Mon R2 IRS 3. In all images north is up and east is to the left.

Upper Left: color representation of our $H$ band speckle image.

Upper Right: color representation of our $K$ band speckle image.

Lower Left: $H+K$ color composite image created from our speckle images.

Lower Right: $K$ band speckle image with annotations.

It is unclear whether the six point objects IRS $3 \mathrm{~A}-\mathrm{F}$ are physically related. The group clearly constitutes a strong local peak in the surface density of infrared sources ${ }^{2}$ and therefore can be considered to constitute a sub-cluster. The IRS 3

2 The six point objects in IRS 3 are all located within 2.6" from IRS 3 A. A much larger 510 square-arcsec area around IRS $3 \mathrm{~A}$ in the $K$ band NICMOS image contains only 10 sources at least as bright as IRS 3 F. Therefore, the surface density of sources around IRS 3 A is 12 times higher than in the surrounding area. sub-cluster seems to be related to a clump in the molecular cloud with a mass of $\sim 30-80 M_{\odot}$ (Henning et al. 1992).

\subsection{Near-infrared photometry}

As our speckle images are not flux calibrated, we used the NICMOS images to obtain photometric measurements of the individual components of IRS 3. Due to the small separations of the individual objects, we had to use small apertures with 
radii of 188 mas (2.5 pixel) for sources $\mathrm{A}, \mathrm{B}, \mathrm{C}, \mathrm{D}$, and $\mathrm{F}$; only for IRS $3 \mathrm{E}$ a larger aperture (500 mas) could be used. The apertures were centered on the $K$ band positions of the individual objects. We employed DAOPHOT in IRAF to measure the fluxes in the apertures and to determine the background flux from a circular point-source free area between IRS 3 B and IRS 3 E. An aperture correction factor for the included-flux difference between 188 mas and 500 mas was determined for IRS 3E with DAOPHOT and then applied to the other sources. Magnitudes in the VEGAMAG system were determined according to the recipe in the NICMOS handbook and are listed in Table 2. Due to the complex structure of the strong diffuse background emission around IRS 3, the accuracy of our photometry is probably not better than about $0.2-0.3$ mag. A comparison of the sum of our $K$ band fluxes for IRS 3A, B, and $\mathrm{C}$, which is $0.69 \mathrm{Jy}$, to the corresponding sum of point-source fluxes derived by Koresko et al. (1993), who found $0.76 \mathrm{Jy}$, shows reasonably good agreement to within $10 \%$.

We constructed a $J-H$ versus $H-K$ color-color diagram (CCD), which is shown in Fig. 2. IRS $3 \mathrm{~A}, \mathrm{~B}$, and $\mathrm{C}$ lie well to the right of the redenning band expected for main sequence colors and therefore show clear evidence for infrared excesses, suggesting the presence of hot circumstellar material, as expected for very young stellar objects. IRS 3 E and D also lie outside the redenning band, but the photometric uncertainties do not allow to draw too strong conclusions from this. IRS $3 \mathrm{~F}$ shows colors consistent with those expected for reddened main-sequence stars without excess; it might either be an unrelated background field star seen through the Mon R2 molecular cloud, or a young star that has already cleared its inner circumstellar environment. However, the non-detection of excess in the $J H K-C C D$ does not necessarily imply that an object has no circumstellar material, because $J, H, K$ observations alone do not cover a sufficient wavelength range to allow complete and unambiguous detection of circumstellar material. Observations at longer wavelengths, e.g. in the $L$ band are much more sensitive to warm dust. Haisch et al. (2001) have demonstrated that $J H K L$ photometry allows an almost unambiguous discrimination between stars with and without circumstellar material. The $L$ band fluxes for IRS 3 A and B can be calculated from the data given in Koresko et al. (1993). For the other components of IRS 3, no $L$ band fluxes are available. The $J-H$ versus $K-L C C D$ is shown in Fig. 2b. Note that many of the Mon R2 infrared sources which lie within the redenning band in the $J H K-\mathrm{CCD}$ clearly show excesses in the $J H K L-C C D$. Also note that IRS $1 \mathrm{SW}$ is rather close to the redenning band in the $J H K-\mathrm{CCD}$, but clearly shows a strong excess in the $J H K L-C C D$. The conclusion we draw from these considerations is as follows: IRS $3 \mathrm{~A}, \mathrm{~B}, \mathrm{C}$, D, and E show evidence for infrared excess, and therefore are most likely YSOs associated to the Mon R2 star forming region. The nature of IRS $3 \mathrm{~F}$ is unclear; it might be an unrelated background object.

The CCD can also be used to estimate the extinction of the objects. For this, we assume intrinsic colors of $(J-H)_{0} \sim 0.6$, what should be roughly appropriate for YSOs, and use the relation $A_{V}=9.37 \quad[(J-H)-0.6]$ (see Rieke \& Lebofsky 1985). The resulting extinctions are listed in Table 2 . We are aware that the extinction estimates are not very reliable for objects with infrared excess, since the excess emission can cause the intrinsic colors of the YSO to deviate from photospheric colors for normal stars. For IRS 3 A, where it is clear that a significant fraction of the emission is scattered light, our extinction estimate should be regarded as a lower limit rather than an accurate value. Extinction determinations for the unresolved IRS $3 \mathrm{~A}+\mathrm{B}+\mathrm{C}$ system arrived at higher values, $A_{V} \sim$ 60-80 mag (Henning et al. 1992; Sellgren et al. 1995). However, these estimates also are not very reliable because they refer to the combined flux of a mixture of individual sources.

Finally, we can derive some information about the masses of the objects from a color-magnitude diagram (Fig. 2c). We have chosen the $J$ band magnitudes and the $J-H$ colors, because these wavelengths are least affected by infrared excesses from circumstellar material. An estimate of the stellar mass can be derived by moving an object backwards along the redenning path until it crosses the color-magnitude relation based on theoretical PMS models for a given age. The presence of an infrared excess, however, can introduce systematic errors and may cause the masses to be overestimated. Especially for IRS $3 \mathrm{~A}$, which is deeply embedded and not directly visible in the $J$ and $H$ bands, this method probably gives only an upper limit to the stellar mass. An additional constraint comes from the total luminosity of $\sim 14000 L_{\odot}$ derived by Henning et al. (1992); this corresponds to an early B-type star and gives an upper mass limit of $<15 M_{\odot}$ (see e.g. Bernasconi \& Maeder 1996). Our final mass estimates are listed in Table 2.

\subsection{Diffuse infrared emission}

IRS $3 \mathrm{~A}$ is surrounded by extended, elongated nebulosity pointing to the south and south-west. A weaker patch of diffuse emission is seen to the north of IRS 3 A. Figure 3 shows a detailed comparison of the $H$ and $K$ band speckle images of IRS 3 A. One can clearly see that the $H$ band emission peak does not coincide with the peak in the $K$ band image but is shifted by 90 mas towards the south-west. We also note that the $H$ band peak is clearly extended and elongated. This implies that in the $H$-band image we do not see the central star directly, but rather a patch of reflected light. The general structure of the nebula around IRS $3 \mathrm{~A}$ is clearly bipolar, suggesting that IRS $3 \mathrm{~A}$ is embedded in a geometrically thick disk or a torus with polar cavities. The difference in brightness between the southern and northern lobes can be understood as an inclination effect: the northern lobe is weaker because it is tilted away from the line-of-sight, while the southern lobe is titled towards the line-of-sight and therefore brighter. A rough, qualitative comparison with radiation transfer simulations (see Sonnhalter et al. 1995) of a star embedded in a torus with polar cavities suggest an inclination angle of about $75^{\circ}$.

IRS 3 B is associated with a strongly elongated nebulous feature pointing to the north-east along a position angle of $50^{\circ}$. This feature is remarkable for three reasons: first, it shows straight, parallel edges, very different from the lobe-like morphology of the emission around IRS 3 A. Second, it seems to consist of three individual knots, the centroids of which are 
Table 2. Positions and magnitudes for the individual components of IRS 3 derived from the NICMOS images. The accuracy of the magnitudes is limited to $\sim 0.2 \mathrm{mag}$. We also list estimates of the extinction and rough mass estimates.

\begin{tabular}{c|ccrrrrrc}
\hline \hline & $\begin{array}{c}\text { RA (J2000) } \\
{[\mathrm{h}: \mathrm{m}: \mathrm{s}]}\end{array}$ & $\begin{array}{c}\text { DEC (J2000) } \\
{[\mathrm{d}: \mathrm{m}: \mathrm{s}]}\end{array}$ & $\begin{array}{r}J \\
{[\mathrm{mag}]}\end{array}$ & $\begin{array}{r}H \\
{[\mathrm{mag}]}\end{array}$ & $\begin{array}{r}K \\
{[\mathrm{mag}]}\end{array}$ & $\begin{array}{c}\text { infrared } \\
\text { excess }\end{array}$ & $\begin{array}{c}A_{V} \\
{[\mathrm{mag}]}\end{array}$ & $\begin{array}{c}M_{\star} \\
{\left[M_{\odot}\right]}\end{array}$ \\
\hline A & $06: 07: 47.836$ & $-06: 22: 56.29$ & 14.6 & 10.8 & 7.9 & Yes & $\gtrsim 30$ & $\sim 12-15$ \\
B & $06: 07: 47.855$ & $-06: 22: 55.49$ & 18.1 & 13.5 & 9.1 & Yes & $\sim 37$ & $\sim 8-12$ \\
C & $06: 07: 47.880$ & $-06: 22: 55.42$ & 15.1 & 11.9 & 9.6 & Yes & $\sim 24$ & $\sim 5-10$ \\
D & $06: 07: 47.885$ & $-06: 22: 54.28$ & 16.8 & 13.7 & 11.8 & Yes(?) & 24 & $\sim 2-5$ \\
E & $06: 07: 47.675$ & $-06: 22: 55.23$ & 15.5 & 13.6 & 12.4 & Yes(?) & 12 & $\sim 1$ \\
F & $06: 07: 47.734$ & $-06: 22: 56.36$ & 17.8 & 15.1 & 13.7 & No & 20 & $\sim 1$ \\
\hline
\end{tabular}
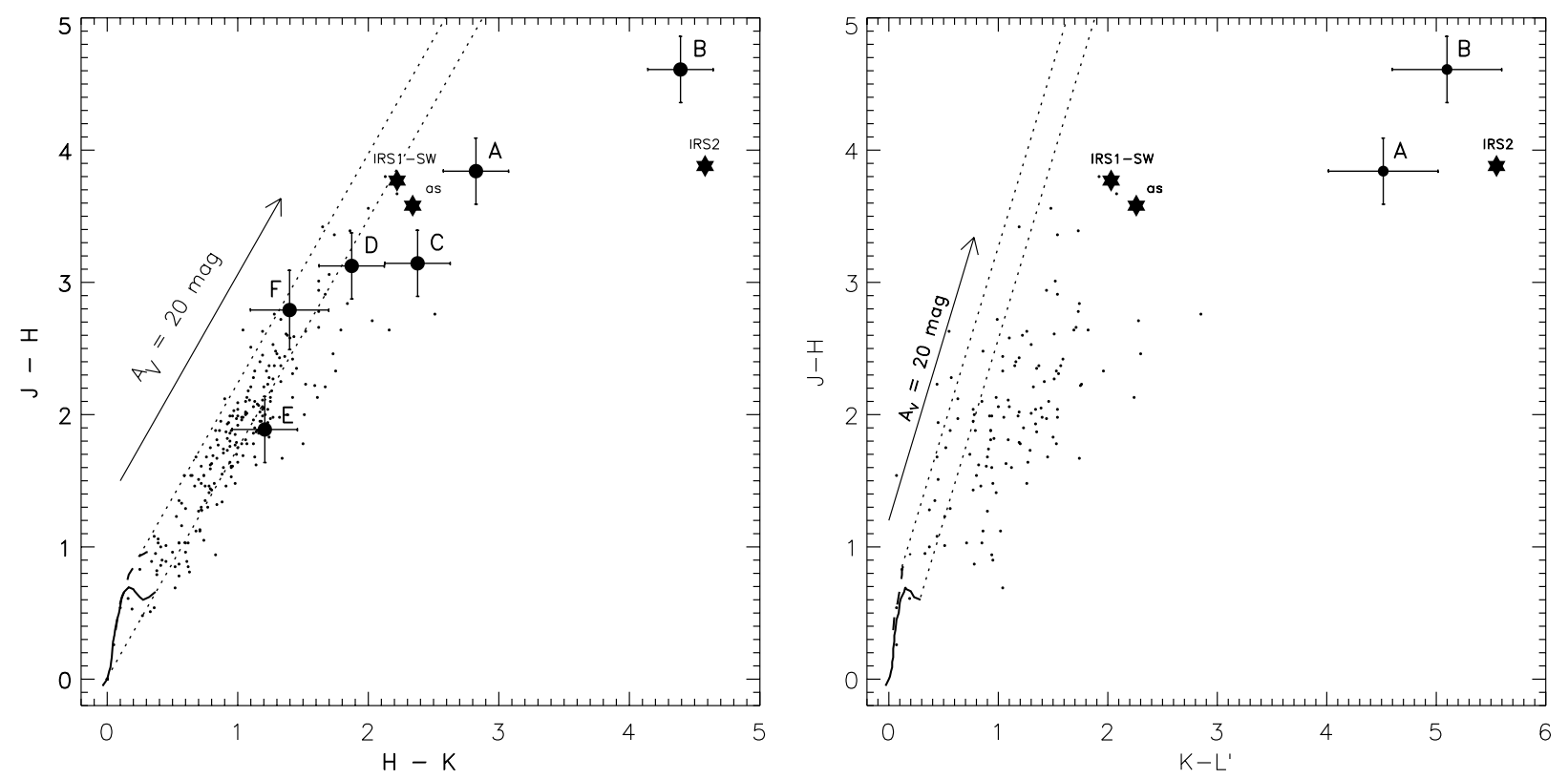

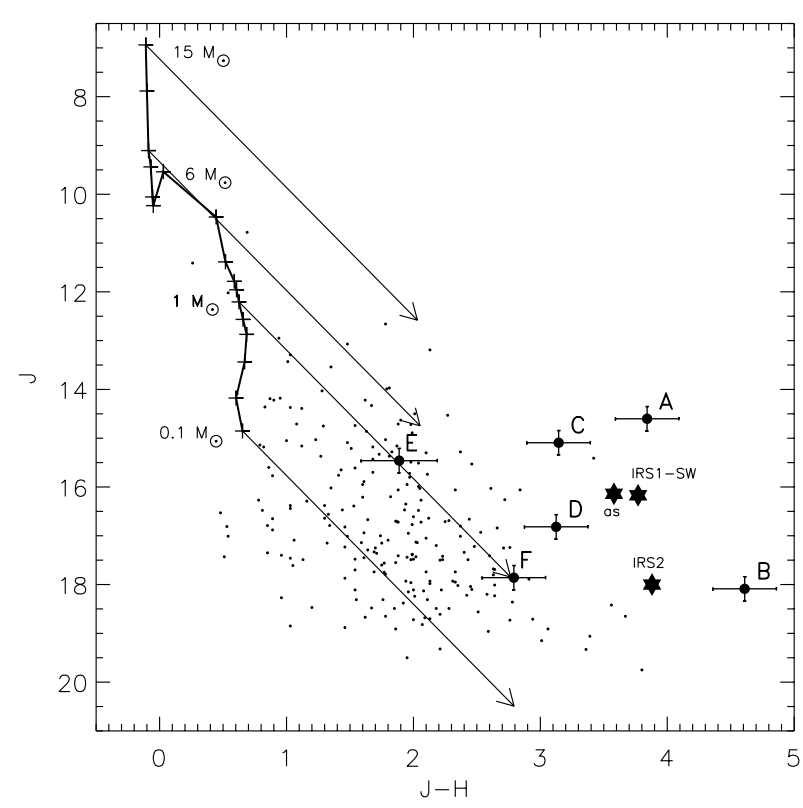

at projected separations of 130,230 , and 290 mas $(\widehat{=} 110,190$, $240 \mathrm{AU})$ from IRS $3 \mathrm{~B}$. Third, the inner two knots are only visible in the $K$ band image, but not in the $H$ band. The third knot is visible in the $H$ band, but is much fainter than in the $K$ band image. This suggests that the emission from these knots is not dominated by scattered light.

All three aspects can be consistently explained by assuming that these knots are related to a jet, or, more generally, material flowing away from IRS 3 B in north-eastern direction. 

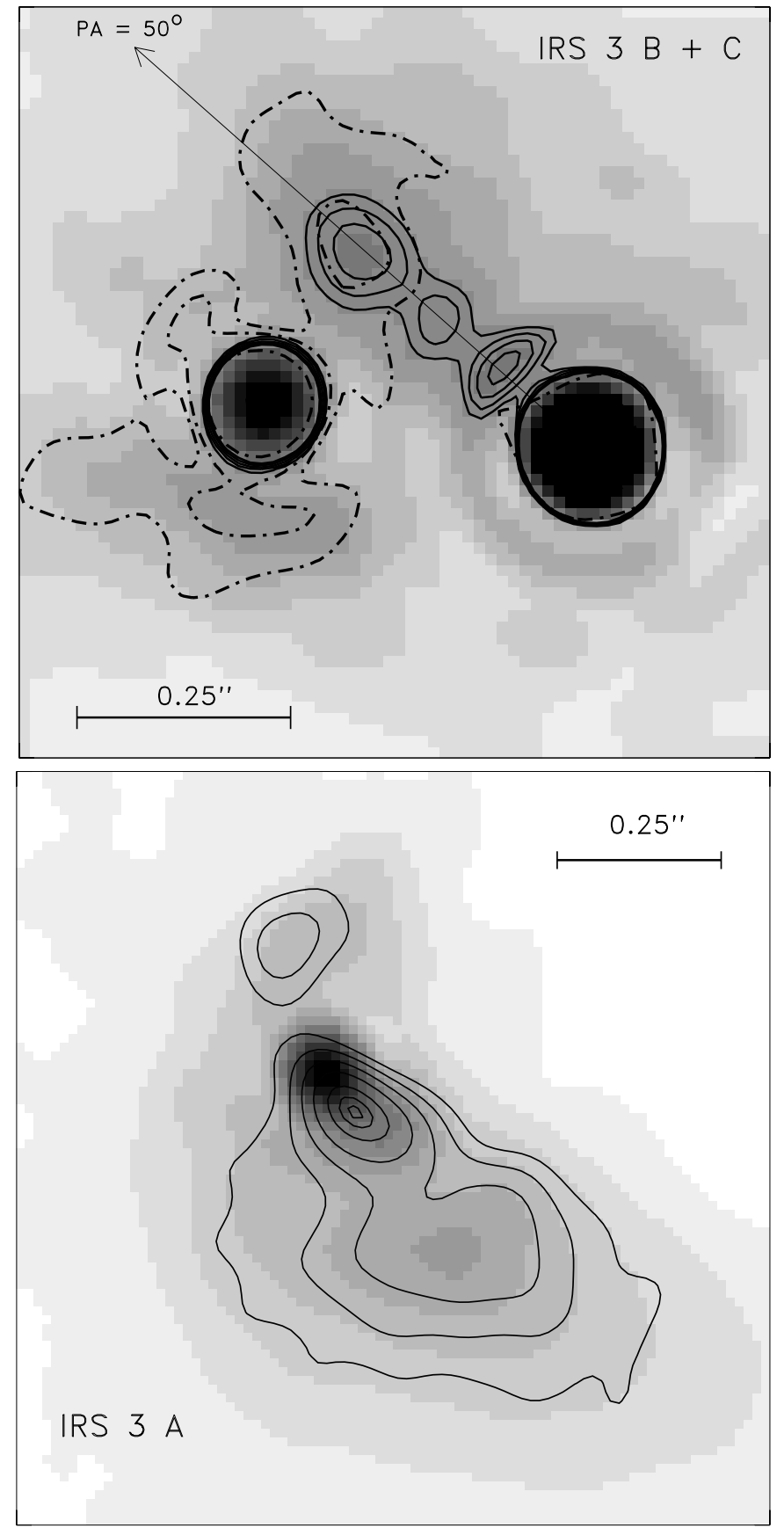

Fig. 3. Upper image: $K$ band speckle image (greyscale + selected thick contours) with dashed-dotted contours of the $H$-band speckle image of Mon R2 IRS 3 B+C.

Lower image: $K$ band speckle image (greyscale) with contours of the $H$ band speckle image of Mon R2 IRS 3 A.

In that case, the $K$ band flux could be dominated by the $2.12 \mu \mathrm{m}$ $v=1-0 \mathrm{~S}(1)$ ro-vibrational emission line of molecular hydrogen, which is a convenient tracer of shocks in jets and outflows (e.g. Smith 1993; Eislöffel et al. 2000). The general shape of the feature and its knot structure is very similar to so-called "microjets" or "small-scale jets" seen at the base of extended outflows from other YSOs (see Dougados et al. 2000; Davis et al. 2002). We measured the width of the knots and found FWHM values of 113 mas (94 AU) for the inner two knots and a $F W H M$ of 135 mas (112 AU) for the outer knot. These values are somewhat larger but still well comparable to the intrinsic jet widths of $\sim 30$ AU found for microjets from T Tauri stars by Dougados et al. (2000). The opening angle is $\leq 40^{\circ}$. A rough estimate of the kinematic age of the knots can also be made: from their projected distance from IRS $3 \mathrm{~B}$, an assumed velocity of $\sim 100 \mathrm{~km} \mathrm{~s}^{-1}$ and inclination angle of $i \sim 45^{\circ}$, we find ages of $\sim 7,13$, and 20 years.

The orientation of the suspected mircojet from IRS 3 B (PA $50^{\circ}$ ) is about perpendicular to the direction of the large-scale molecular outflow, which has a position angle of $135^{\circ} / 315^{\circ}$. This suggests that the suspected mircojet is not related to this old large-scale outflow. It might, however, well be related to the compact high-velocity gas associated with IRS 3 (Giannakopoulou et al. 1997), i.e. the younger outflow, which has a roughly orthogonal orientation with respect to the older large-scale outflow (Meyers-Rice \& Lada 1991).

Finally, we note a curved arc of emission to the north of IRS 3 B. The NICMOS image shows this arc to extend at least $3^{\prime \prime}$ in north-eastern direction and the large-scale diffuse emission associated to IRS 3 is also elongated in $50^{\circ}$ direction and can be traced up to distances of $\sim 35^{\prime \prime}$ (see e.g. Fig. 1 in Yao et al. 1997). This material might be associated with the outflow from IRS $3 \mathrm{~B}$. The kinematic age of the outermost parts would be about 240 years.

\section{X-ray emission from IRS 3}

Very little is known today about the X-ray activity of extremely young intermediate- and high-mass stars. X-ray observations of massive YSOs did either detect no X-ray emission at all or failed to provide clear identifications of the X-ray source with the massive YSO (see e.g. Garmire et al. 2000 for the case of the BN object Orion). Gregorio-Hetem et al. (1998) presented the first X-ray observation of the Mon R2 star forming region with the ROSAT PSPC. They detected faint X-ray emission from a position close to IRS 3 , but the positional uncertainty of $\pm 15^{\prime \prime}$ prevented a reliable identification of the source. Kohno et al. (2002) recently observed the Mon R2 star forming region with the Chandra X-ray observatory. They found more than $150 \mathrm{X}$-ray sources, and interestingly detected X-ray emission from the intermediate/high-mass YSOs IRS 1, IRS 2, and IRS 3. This provided the first reliable detection of hard X-ray emission from very young intermediate/high-mass stars. With the new information on the YSOs in IRS 3 derived from our high-resolution infrared study, it is worthwhile to take a new and detailed look at the X-ray data for IRS 3.

\subsection{X-ray source identification}

We analyzed the data with the CIAO 2.1.2 software package ${ }^{3}$ provided by the Chandra X-ray Center and used the Level 2 processed event list provided by the pipeline processing at the Chandra X-ray Center. In our analysis we excluded all events with energies above $10 \mathrm{keV}$, because essentially all of these are caused by charged particle background. IRS 3 is located close

\footnotetext{
${ }^{3}$ http://asc.harvard.edu/ciao/download.html
} 


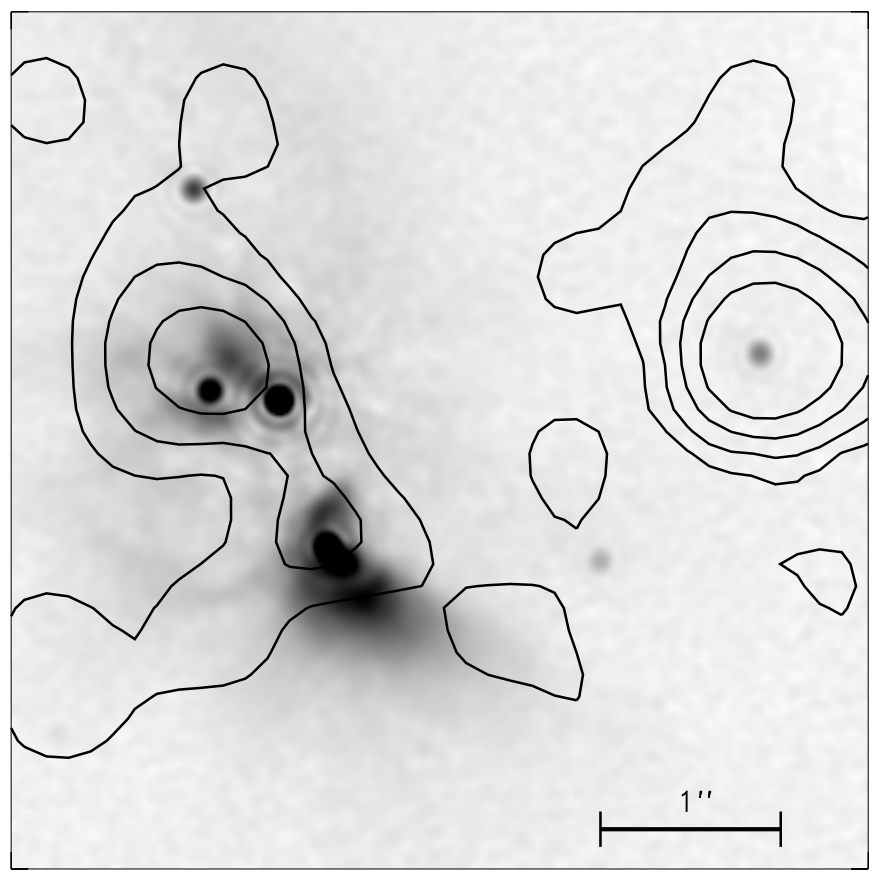

Fig. 4. Greyscale representation of our $K$ band speckle image of Mon R2 IRS 3 with contour lines of the Chandra X-ray image.

to the aimpoint of the observation, where ACIS provides subarcsecond resolution (the on-axis half energy radius of the PSF is $0.42^{\prime \prime}$ ). The combination of the superb point-spread function of Chandra /ACIS and the large number of X-ray sources in this image of Mon R2 allows a very reliable source identification. After correcting for a small systematic aspect error (see also Kohno et al. 2002) the final accuracy of the aspect solution is better than $\sim 0.5^{\prime \prime}$. In Fig. 4 we plot the contours of the Chandra X-ray image ${ }^{4}$ on our $K$ band speckle image. The strongest X-ray peak coincides with IRS $3 \mathrm{E}$ and contains 60 photons. A much weaker peak is found at the position of IRS 3 C, and another weak peak at IRS 3 A. In order to check the significance of the two weaker peaks we investigated the $\mathrm{X}$-ray background in the area around IRS 3 and found a mean background level of $(0.06 \pm 0.01)$ counts/pixel. The single ACIS pixel coinciding with the position of IRS 3 A contains 5 photons. As the Poisson probability to find 5 or more counts per pixel as a result of a background fluctuation would be only $6.2 \times 10^{-9}$, this represents an highly significant X-ray detection. The pixel at the position of IRS $3 \mathrm{C}$ contains 8 photons, i.e. the X-ray detection of this YSOs is also beyond question. Since the separation of the sources IRS 3 A-C is similar or even smaller than the ACIS pixel size, a clear identification of the $\mathrm{X}$-ray emission peaks with individual infrared sources is not trivial. Fortunately, the relatively strong X-ray emission from IRS 3 E provides a good astrometric reference and allows a reliable identification of the two weak peaks with IRS 3 A and C. To check whether some fraction of the X-ray flux we associate to IRS $3 \mathrm{C}$ might actually originate from IRS 3 B, we simulated

\footnotetext{
${ }^{4}$ To create this image we reprocessed the level 1 event file and removed the \pm 0.5 pixel randomization, which is applied in standard data processing. This yields a slight improvement in the overall PSF.
}
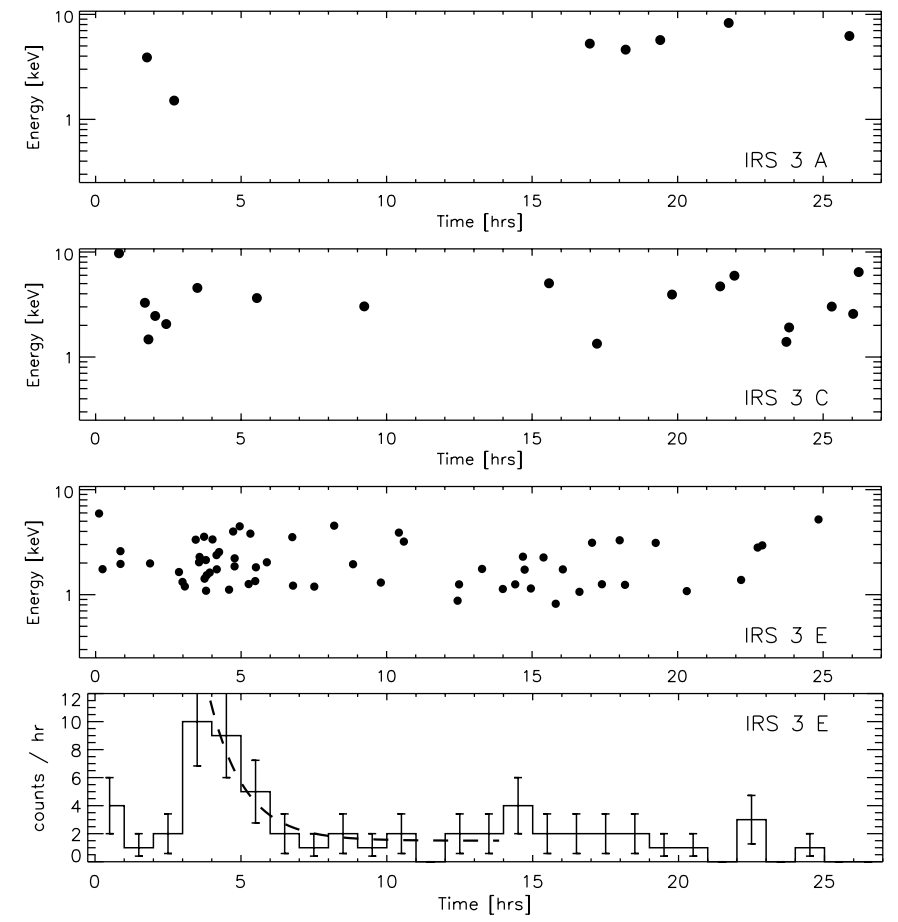

Fig. 5. The upper three plots show the photon arrival times and energies for the sources IRS 3 A, IRS 3 C, and IRS 3 E. The lower plot shows the histogram for the count rate of IRS $3 \mathrm{E}$.

images with the MARX Chandra data simulator ${ }^{5}$. These simulations show that the observed photon distribution is fully consistent with the assumption that only IRS $3 \mathrm{C}$ is an X-ray source and there is no contribution from IRS $3 \mathrm{~B}$. If IRS $3 \mathrm{~B}$ would be a similarly strong source than IRS $3 \mathrm{C}$, then the resulting photon distribution should be clearly elongated in east-west direction, what is not observed.

\subsection{X-ray properties of IRS $3 A, C$, and $E$}

We extracted the photons from non-overlapping source regions with radii of $0.5^{\prime \prime}$ centered at the infrared positions of IRS 3 A and $\mathrm{C}$, and a $1^{\prime \prime}$ radius source region for IRS $3 \mathrm{E}$. We derived an aperture correction factor by analyzing the PSFs of several nearby X-ray sources and determined the background in an annulus around IRS 3 . The background-subtracted source count rates are listed in Table 3.

In Fig. 5 we plot the energies and arrival times for the individual photons detected from each source. First we consider the temporal variability. The photon arrival times for IRS 3 A show a 14 hour period without any detected photon, while the following 10 hour period contains 5 photons. A Poissonstatistical evaluation (see Preibisch \& Zinnecker 2002) gives a probability of only $10 \%$ that this is the result of a random fluctuation; the probability for temporal variability of the X-ray flux from IRS $3 \mathrm{~A}$ is therefore $90 \%$. For IRS $3 \mathrm{C}$ we find a conspicuous accumulation of photons in the second and third hour of the observation. A Poisson-statistical evaluation gives a

\footnotetext{
5 For further information on MARX see http://space.mit.edu/CXC/MARX/.
} 
Table 3. X-ray results. This table lists the background-subtracted source count rates, the probability of variability (PoV) based on Poisson statistics, the median energy $(\widetilde{E})$ of the detected photons, the hydrogen column density assumed for the spectral fitting, the fitted plasma temperature, the resulting extinction-corrected X-ray luminosity in the $0.2-10 \mathrm{keV}$ band, and the fractional X-ray luminosity.

\begin{tabular}{l|ccccccc}
\hline \hline IRS 3 & $\begin{array}{c}\text { count rate } \\
{[\mathrm{cnts} / \mathrm{ksec}]}\end{array}$ & PoV & $\begin{array}{c}\widetilde{E} \\
{[\mathrm{keV}]}\end{array}$ & $\begin{array}{c}N_{\mathrm{H}} \\
{\left[10^{22} \mathrm{~cm}^{-2}\right]}\end{array}$ & $\begin{array}{c}k T \\
{[\mathrm{keV}]}\end{array}$ & $\begin{array}{c}L_{\mathrm{X}} \\
{\left[10^{30} \mathrm{erg} / \mathrm{sec}\right]}\end{array}$ & $\log \left(L_{\mathrm{X}} / L_{\text {bol }}\right)$ \\
\hline $\mathrm{A}$ & $0.166 \pm 0.041$ & $90 \%$ & 5.3 & $\gtrsim 6.0$ & $>4$ & $\approx 0.8$ & $\sim-7.7$ \\
$\mathrm{C}$ & $0.435 \pm 0.133$ & $98.4 \%$ & 3.3 & 4.8 & $4[2.5-6]$ & 1.1 & $\sim-7.0$ \\
$\mathrm{E}$ & $0.759 \pm 0.087$ & $>99.99 \%$ & 1.8 & 2.4 & $2.5[2-3]$ & 1.5 & -3.7 \\
\hline
\end{tabular}

probability of only $1.6 \%$ that this is a random fluctuation, i.e. it clearly indicates variability. Finally, IRS 3 E is obviously variable; the Poisson-statistical probability for a random fluctuation is only $10^{-5}$. The relatively large number of photons detected from IRS 3 E allows us to construct a lightcurve, which shows a flare that occurred about 3 hours after the start of the observation. The dashed line shows a simple fit to the observed light-curve, assuming a flare amplitude of a factor of 6.7 and an exponential decay time of 1.2 hours. This resembles the typical properties of flares on young coronally active stars (see e.g. Preibisch \& Zinnecker 2002).

Next we consider the distributions of X-ray photon energies. Generally, only photons with energies above $\sim 1 \mathrm{keV}$ are detected; this is primarily the consequence of the strong extinction, which efficiently absorbs the softer photons. The median photon energies are $5.3 \mathrm{keV}$ for IRS $3 \mathrm{~A}$ and $3.3 \mathrm{keV}$ for IRS $3 \mathrm{C}$, while IRS $3 \mathrm{E}$ has an obviously softer X-ray spectrum with a median value of $1.8 \mathrm{keV}$. To get information of the plasma temperatures, we used the CIAO thread "psextract" to extract the individual pulse-hight spectra and to build the corresponding individual redistribution matrix files and ancillary response files for each source and then fitted the ungrouped pulse-height spectra with XSPEC. We used the XSPEC model "raymond" for the emission from a thermal plasma and "wabs" for the absorption model. The X-ray absorption is a function of the hydrogen column density, which can be computed from the visual extinction according to the relation $N_{\mathrm{H}} \sim A_{V} \times 2 \times 10^{21} \mathrm{~cm}^{-2}$ (Ryter 1996). We used the column densities listed in Table 3.

For IRS $3 \mathrm{E}$ the plasma temperature is well constrained to $2.5 \pm 0.5 \mathrm{keV}$, a value typical for coronally active YSOs (see e.g. Preibisch \& Zinnecker 2002). For IRS 3 C we find a temperature of about $4 \mathrm{keV}$. In the case of IRS 3 A the low number of detected photons does not allow us to tightly constrain the plasma temperature. The spectrum is clearly very hard, with most of the detected photons having energies above $5 \mathrm{keV}$. Detailed simulations of pulse-hight spectra with XSPEC show that such a hard spectrum requires very high plasma temperatures, probably above $k T \sim 8 \mathrm{keV}$. Due to the uncertainty in the extinction towards IRS $3 \mathrm{~A}$, which might perhaps be considerably larger than the $A_{V} \sim 30$ mag suggested by the nearinfrared colors (see discussion above), we conservatively estimate a lower limit to the plasma temperature of $k T>4 \mathrm{keV}$.

Finally, we computed the extinction-corrected X-ray luminosities from the fitting results. All results are summarized in Table 3. We note that the X-ray properties we find for IRS 3 A and $\mathrm{C}$ are very similar to those determined by Kohno et al. (2002) for the other intermediate/high-mass YSOs in Mon R2, IRS $1 \mathrm{SW}$, IRS 2, and $a_{\mathrm{s}}$.

\subsection{The origin of the $X$-ray emission}

Stellar X-ray emission is usually explained by one of two different mechanisms, which operate in different mass regimes. Low-mass stars $\left(M_{\star} \leq 2 M_{\odot}\right)$ have outer convection zones, and a stellar dynamo leads to the formation of a corona in which hot $\left(>10^{6} \mathrm{~K}\right)$ plasma is magnetically confined. In comparison to the Sun, young low-mass stars often exhibit highly elevated levels of coronal emission; typical fractional X-ray luminosities and plasma temperatures for T Tauri stars are $L_{\mathrm{X}} / L_{\mathrm{bol}} \sim$ $10^{-4}-10^{-3}$ and $k T \sim 0.5-2 \mathrm{keV}$; strong temporal variability and large flares are often observed (for a review see Feigelson \& Montmerle 1999). Several extremely young ( $\$ 10^{5}$ yrs) lowmass YSOs (Class I protostars) have been found to emit very hard X-rays $(k T \sim 3-8 \mathrm{keV})$, which is though to be related to magnetic interactions between the protostar and its circumstellar material (Feigelson \& Montmerle 1999).

High-mass stars $\left(M_{\star} \geq 10 M_{\odot}\right)$, on the other hand, have fast radiation-driven stellar winds. Instabilities lead to strong internal wind shocks in which material is heated to X-ray emitting temperatures. Typical fractional $\mathrm{X}$-ray luminosities are $L_{\mathrm{X}} / L_{\mathrm{bol}} \sim 10^{-7}$, the X-ray emission shows very little variability and a rather soft X-ray spectrum with typical temperatures of $k T \lesssim 0.5 \mathrm{keV}$ (see Berghöfer et al. 1997).

In Fig. 6 we plot the X-ray plasma temperatures of the intermediate/high-mass YSOs in R Mon against their X-ray luminosities, and compare them to those of $\mathrm{T}$ Tauri stars in IC 348, class I protostars in different star forming regions, and early B-type main-sequence stars. The X-ray properties of IRS $3 \mathrm{E}$, which is presumably a low-mass YSO, are very similar to those of coronally active young low-mass stars.

The X-ray properties of the intermediate/high-mass YSOs IRS $3 \mathrm{~A}$ and $\mathrm{C}$, which are very similar to those of the other Mon R2 intermediate/high-mass YSOs IRS 1 SW, IRS 2, and $a_{\mathrm{s}}$ (Kohno et al. 2002), cannot, however, be explained by the standard scenario for massive stars. Their emission is clearly much harder than the prediction of stellar wind models (e.g. Krolik \& Raymond 1985). The observed plasma temperatures of $\gtrsim 4 \mathrm{keV}$ $\left(\gtrsim 50 \times 10^{6} \mathrm{~K}\right)$ are ten times higher than plasma temperatures typical for early B-type main-sequence stars (see Cohen et al. 1997). Also, their lightcurves suggest strong temporal variability. Thus, in accordance with Kohno et al. (2002), we conclude 


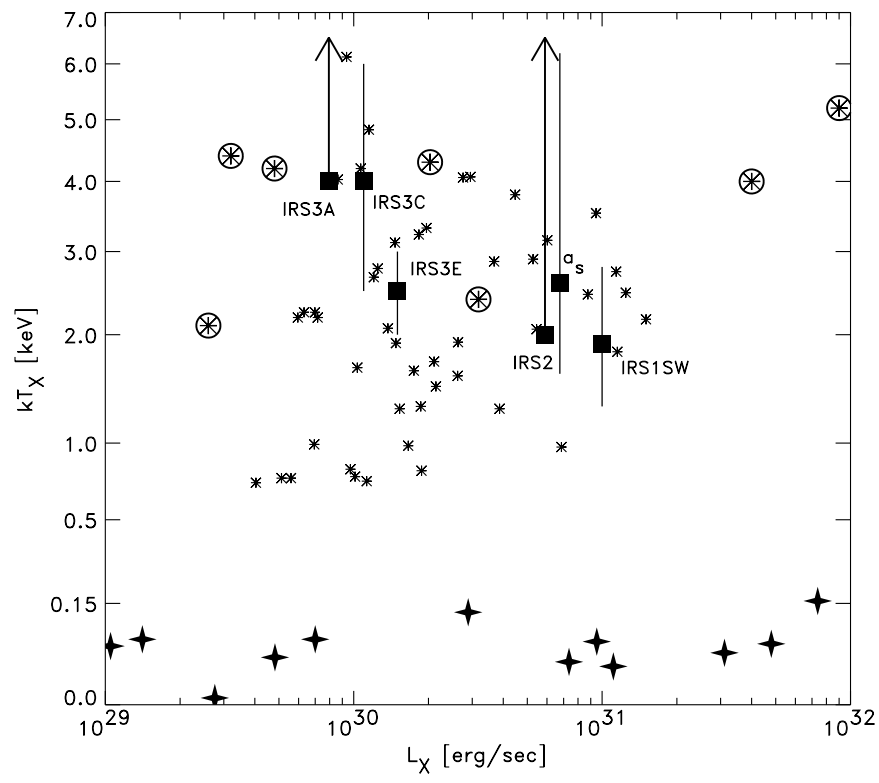

Fig. 6. The plot shows the X-ray plasma temperature versus X-ray luminosity for the Mon R2 X-ray sources and several types of (young) stars. IRS 3 A, IRS 3 C, and IRS 3 E are shown as solid squares. We also show the data for other massive/intermediate-mass YSOs IRS 1 SW, IRS 2, and $a_{\mathrm{s}}$ in Mon R2 taken from Kohno et al. (2002). The big solid asterisks show data for early B-type main-sequence stars from Cohen et al. (1997; temperature from isothermal model fitting, their Table 7). The small asterisks show data for T Tauri stars in the young cluster IC 348 from Preibisch \& Zinnecker (2002). The encircled small asterisks show data for X-ray detected low-mass class I protostars from Ozawa et al. (1999), Tsuboi et al. (2000), and Imanishi et al. (2001).

that the X-ray properties of the intermediate/high-mass YSOs in Mon R2 (IRS 1 SW, IRS 2, $a_{\mathrm{s}}$, and IRS $3 \mathrm{~A}$ and C) clearly differ from those of $\gtrsim 10^{6}$ yrs old $\mathrm{B}$ stars and that the wind shock model is not able to account for a substantial fraction of their observed X-ray emission. The intermediate/high-mass YSOs also cannot have a solar-like dynamo, and therefore are not expected to have an X-ray emitting stellar corona. Their $\mathrm{X}$-ray properties are, however, similar to those observed for low-mass class I protostars. The hard and often strongly variable X-ray emission from these very young embedded lowmass YSOs is probably caused by magnetic interactions between the protostar and its accretion disk (see Feigelson \& Montmerle 1999; Montmerle et al. 2000). This suggests that a similar mechanism is operating in the intermediate/high-mass YSOs in R Mon and therefore supports the idea that these intermediate/high-mass YSOs have circumstellar disks.

\section{Summary and conclusions}

Our high-resolution study of the infrared source Mon R2 IRS 3 resolves this object into three individual stellar sources, all of which display near-infrared excess emission, and thus are YSOs. In addition, our images show at least three further sources, which are up to $6 \mathrm{mag}$ fainter than the brightest object. The brightest and presumably most massive object, IRS $3 \mathrm{~A}$, is surrounded by a fan-shaped bipolar nebula and therefore seems to be embedded in a dense circumstellar torus or a thick disk. We discovered an elongated structure next to IRS 3 B, which seems to be a micro-jet, consisting of three individual knots at projected separations of 130, 230, and 290 mas (110, 190, and $240 \mathrm{AU}$ ) from IRS $3 \mathrm{~B}$. This suggests that IRS $3 \mathrm{~B}$ is the source of the high-velocity molecular outflow observed from Mon R2 IRS 3. As the kinematic age of the supposed jet knots is of the order of only a few years, repeated high-resolution observations should soon reveal motion of these knots if our interpretation is correct.

Our analysis of Chandra archive X-ray data shows that the intermediate/high-mass YSOs IRS $3 \mathrm{~A}$ and IRS $3 \mathrm{C}$ are sources of hard and variable X-ray emission. The best explanation for the observed $\mathrm{X}$-ray properties is the model of magnetic interaction between a YSO and its surrounding disks originally proposed for low-mass class I protostars. An important conclusion from this is that the observed $X$-ray emission provides indirect evidence for the existence of disks around these intermediate/high-mass YSOs. This is interesting, because the formation mechanism of massive stars is still not well understood (cf. Stahler et al. 2000). The well established scenario of gravitational collapse and subsequent accretion for low-mass star formation faces serious difficulties in the case of highmass stars, because as soon as the stellar core reaches a mass of $\sim 8-10 M_{\odot}$, the radiation pressure on the infalling dust strongly opposes the accretion and thus limits the mass of the forming star (e.g. Yorke \& Krügel 1977). Therefore, it has been argued that massive stars cannot form via disk accretion and alternative models, like collisions and coagulation of protostars in dense forming stellar clusters (see Bonnell et al. 1998) have been suggested. Recent theoretical studies, however, have shown that massive stars can in principle be formed via accretion through a disk if the anisotropy of the protostellar radiation field and the frequency dependence of the dust properties are properly taken into account Yorke \& Sonnhalter (2002). The observed X-ray properties support the disk-accretion scenario.

Acknowledgements. We are grateful to the referee, C. Koresko, for his very constructive referee's report which helped to improve this paper.

\section{References}

Alvarez, C. A., \& Hoare, M. G. 2002, in The Earliest Phases of Massive Star Birth (Third Boulder Hot Star Workshop), ed. P. A. Crowther, ASP Conf. Ser., 267, 335

Aspin, C., \& Walther, D. M. 1990, A\&A, 235, 387

Beckwith, S., Evans, N. J. II, Becklin, E. E., \& Neugebauer, G. 1976, ApJ, 208, 390

Berghöfer, T. W., Schmitt, J. H. M. M., Danner, R., \& Cassinelli, J. P. 1997, A\&A, 322, 167

Bernasconi, P. A., \& Maeder, A. 1996, A\&A, 307, 829

Bonnell, I. A., Bate, M. R., \& Zinnecker, H. 1998, MNRAS, 298, 93

Carpenter, J. M., Meyer, M. R., Dougados, C., Strom, S. E., \& Hillenbrand, L. A. 1997, AJ, 114, 198

Cohen, D. H., Cassinelli, J. P., \& MacFarlane, J. J. 1997, ApJ, 487, 867

Davis, C. J., Stern, L., Ray, T. P., \& Chrysostomou, A. 2002, A\&A, 382,1021

Dougados, C., Cabrit, S., Lavalley, C., \& Menard, F. 2000, A\&A, 367, L61 
Eislöffel, J., Smith, M. D., Davis, C. J., \& Ray, T. P. 1996, AJ, 112, 2086

Eislöffel, J., Mundt, R., Ray, T. P., \& Rodriguez, L. F. 2000, in Protostars and Planets IV, ed. V. Mannings, A. P. Boss, \& S. S. Russel (Tucson: University of Arizona Press), 815

Feigelson, E. D., \& Montmerle, T. 1999, ARA\&A, 37, 363

Garmire, G., Feigelson, E. D., Broos, P., et al. 2000, AJ, 120, 1426

Giannakopoulou, J., Mitchell, G. F., Hasegawa, T. I., Matthews, H. E., \& Maillard, J.-P. 1997, ApJ, 487, 346

Gregorio-Hetem J., Montmerle T., Casanova S., Feigelson E.D., 1998, 331,193

Haisch, K. E., Lada, E. A., Pina, R. K., Telesco, C. M., \& Lada, C. J. 2001, AJ, 121, 1512

Henning, Th., Chini, R., \& Pfau, W. 1992, A\&A, 263, 285

Herbst, W., \& Racine, R. 1976, AJ, 81, 840

Herbig, G. H. 1960, ApJS, 4, 337

Hofmann, K.-H., \& Weigelt, G. 1986, A\&A, 167, L15

Howard, E. M., Pipher, J. L., \& Forrest, W. J. 1994, ApJ, 425, 707

Kohno, M., Koyama, K., \& Hamaguchi, K. 2002, ApJ, 576, 423

Koresko, C. D., Beckwith, S., Ghez, A. M., Matthews, K., \& Smith, D. A. 1993, AJ, 105, 1481

Krolik, J. H., \& Raymond, J. C. 1985, ApJ, 298, 660

Labeyrie, A. 1970, A\&A, 6, 85

Lohmann, A. W., Weigelt, G., \& Wirnitzer, B. 1983, Appl. Opt., 22, 4028
Massi, M., Felli, M., \& Simon, M. 1985, A\&A, 152, 387

McCarthy, D. W. 1982, ApJ, 257, L93

McCaughrean, M. J., Zinnecker, H., \& Rayner, J. T. 1994, ApJ, 436, L189

Montmerle, T., Grosso, N., \& Koyama, K. 2000, ApJ, 532, 1097

Ozawa, H., Nagase, F., Ueda, Y., Dotani, T., \& Ishida, M. 1999, ApJ, 523, L81

Preibisch, Th., \& Zinnecker, H. 2002, AJ, 123, 1613

Rieke, G. H., \& Lebofsky, M. J. 1985, ApJ, 288, 618

Ryter, C. E., Ap\&SS, 236, 285

Sellgren, K., Brooke, T. Y., Smith, R. G., \& Geballe, T. R. 1995, ApJ, 449, L69

Smith, M. D. 1993, ApJ, 406, 520

Sonnhalter, C., Preibisch, Th., \& Yorke, H. W. 1995, A\&A, 299, 545

Stahler, S. W., Palla, F., \& Ho, P. T. P. 2000, in Protostars and Planets IV, ed. V. Mannings, A. Boss, \& S. Russel (University of Arizona Press), 327

Tsuboi, Y., Imanishi, K., Koyama, K., Grosso, N., \& Montmerle, T. 2000, ApJ, 532, 1089

Weigelt, G. 1977, Opt. Commun., 21, 55

Weigelt, G. 1991, in Progress in Optics, vol. 29, ed. E. Wolf (Elsevier Science Publishers), 295

Yao, Y., Hirata, N., Ishi, M., et al. 1997, ApJ, 490, 281

Yorke, H. W., \& Sonnhalter, C. 2002, ApJ, 569, 846 